\title{
Profesorado en la detección de actitudes adultas pro-consumo de drogas y su efecto en menores
}

\author{
María Lidia Platas-Ferreiro \\ Departamento de Didáctica y Organización escolar, Universidad de Santiago de Compostela, España
}

\begin{abstract}
Resumen
La familia constituye el núcleo donde se fraguan los modelos y pautas a seguir por sus miembros, por lo que debería constituir un espacio privilegiado para los hijos/as en lo relacionado con la prevención del consumo de drogas, fomentando la comunicación, el respeto y un estilo de vida saludable. En ocasiones, cuando la familia no constituye un modelo adecuado para el/la menor, queda en manos del profesorado detectar esta problemática de modelo familiar pro-consumo e intervenir para prevenir conductas de riesgo por parte del alumnado.
\end{abstract}

Palabras clave: [menores, familia, profesorado, consumo, drogas.]

Describir las relaciones entre el consumo de drogas en el ámbito familiar y el de los/as hijos/as menores analizando cómo la familia influye y determina el consumo y la actitud ante las drogas en sus propios hijos, es una tarea donde el profesorado puede convertirse en mediador tanto para prevenir como para intervenir cuando sea preciso.

\section{La fuerza de la mediación}

Los programas de mediación escolar fomentan la capacidad de trabajar unidos contra los problemas, no contra las personas. Esto no sólo comporta ventajas evidentes en cuanto a la prevención e intervención frente a los conflictos, sino también posibilidades innovadoras y trasnformadoras del propio contexto educativo. La mediación no daña, al contrario, posee una función curativa, ya que reconoce las heridas sin necesidad de abrirlas (Ury, 2000).

Cuando los conflictos se median, la agresividad se canaliza de forma constructiva. (Boqué, 2003). Los protagonistas del conflicto aprenden estrategias positivas y efectivas de relación interpersonal, de regulación, de canalización, y de expresión de sentimientos, emociones.., que les permiten el autocontrol y autogestionar por si mismos la mayoría de los problemas y dificultades que se van a encontrar tanto dentro como fuera del centro educativo.

La mediación, como señalan Hartman y Chesley (1997) repercute en la transformación del sistema educativo, en una mayor implicación de los diferentes sectores de la comunidad educativa, en la comprensión y aceptación de la diversidad.

\section{El consumo en el ámbito familiar con hijos menores}

Los datos recogidos en estudios sociológicos anteriores, llevados a cabo también en la capital lucense, nos llevan a asociar casi sin pensar, el tipo de ocio familiar con las posibilidades de consumo de los hijos, ya que, prácticamente el $90 \%$ de los padres y madres, ocupan su tiempo libre en pasear, tomar algo, visitar a alguien e ir a un centro comercial (Platas, 2009).

Por otra parte, se encontró que el 85\% de los alumnos y alumnas de 10 a 12 años, estarían dispuestos a hablar a sus seres más queridos (familia y amistades) de los peligros del consumo de droga (Platas, 2012).

Siguiendo con datos lucenses, en un estudio realizado por Platas (2010) sobre el estado de la situación por consumo de drogas, de los 820 menores de $5^{\circ}$ y $6^{\circ}$ de primaria encuestados/as, 1 de cada 4 menores tienen un padre/madre fumador $\mathrm{y}$, no parece que la casualidad justifique que, absolutamente todos los menores que han probado el tabaco antes de los 12 años, tengan familiares que viven con ellos que fuman.

Platas (2012) señala en el II Plan Municipal de Drogodependencias, que el consumo de porros o cocaína dentro de la vivienda familiar apenas es apreciable. Sin embargo, sí se puede constatar que los padres y madres acostumbran a consumir alcohol en mayor medida que el tabaco, dentro del hogar y a la vista de sus hijos e hijas. Resulta significativo que quienes fuman en casa, lo hacen incluso cuando el consumo es bastante alto, lo que nos lleva a un $25 \%$ de padres y madres fumadoras que lo hacen en presencia de sus hijos e hijas menores. Por otra parte, el alcohol se consume dentro del hogar con mucha mayor frecuencia ( 1 de cada 2 padres y casi 1 de cada 3 madres!) pero sobre todo en dosis bajas. (Véase Tabla 1)

Tabla 1.

Consumo parental en la vivienda familiar

\begin{tabular}{|l|c|c|c|c|c|c|c|c|}
\hline \multirow{2}{*}{} & \multicolumn{2}{|c|}{$\begin{array}{c}\text { Alcohol } \\
(\%)\end{array}$} & \multicolumn{2}{c|}{$\begin{array}{c}\text { Porros } \\
(\%)\end{array}$} & \multicolumn{2}{c|}{$\begin{array}{c}\text { Cocaína } \\
(\%)\end{array}$} & \multicolumn{2}{|c|}{$\begin{array}{c}\text { Tabaco } \\
(\%)\end{array}$} \\
\cline { 2 - 10 } & $\begin{array}{c}\text { Ma } \\
\text { dre }\end{array}$ & $\begin{array}{c}\text { Pa } \\
\text { dre }\end{array}$ & $\begin{array}{c}\text { Ma } \\
\text { dre }\end{array}$ & $\begin{array}{c}\text { Pa } \\
\text { dre }\end{array}$ & $\begin{array}{c}\text { Ma } \\
\text { dre }\end{array}$ & $\begin{array}{c}\text { Pa } \\
\text { dre }\end{array}$ & $\begin{array}{c}\text { Ma } \\
\text { dre }\end{array}$ & $\begin{array}{c}\text { Pa } \\
\text { dre }\end{array}$ \\
\hline Nada & 7,6 & 4,0 & 8,6 & 8,5 & 8,7 & 8,6 & 6,7 & 5,2 \\
\hline Poco & 33,3 & 51,8 & 0,3 & 0,7 & 0,0 & 0,2 & 12,5 & 14,9 \\
\hline Bastante & 1,4 & 6,6 & 0,1 & 0,2 & 0,0 & 0,1 & 11,3 & 12,9 \\
\hline Mucho & 0,1 & 1,3 & 0,2 & 0,3 & 0,1 & 0,1 & 5,2 & 6,9 \\
\hline
\end{tabular}


Por tanto, alcohol y tabaco, son asuntos en los que debemos trabajar con las familias, enfatizando su papel como "modelos perjudiciales de consumo" para sus hijas e hijas, siendo éste uno de los objetivos del Plan Nacional sobre Drogas y del Plan Municipal de Lugp sobre Drogodependencias durante el período 20122016.

\section{Objetivos}

Describir las relaciones entre el consumo de drogas en el ámbito familiar y el de los hijos menores analizando cómo la familia influye y determina el consumo y la actitud ante las drogas en sus propios hijos, es una tarea donde el profesorado puede convertirse en mediador tanto para prevenir como para intervenir cuando sea preciso.

\section{Método}

Estudiantes de Primaria de la capital lucense que han respondido a una encuesta que pretendía recoger información sobre los hábitos de consumo, la actitud y el conocimientos que estos menores tienen hacia las drogas en general y cómo la actitud, el conocimiento y el consumo de sus padres y madres pueden influirles de una forma más o menos determinante.

\section{Participantes}

822 estudiantes de la capital lucense (398 niñas y 414 niños) que cursan $5^{\circ}$ (373) y/o $6^{\circ}$ (449) de primaria, todos entre 10 y 12 años de edad.

24 colegios que ofertan Educación primaria en la capital lucense; esta cantidad se corresponde con el 100\% de centros educativos. (Véase Tabla 2)

Tabla 2.

Colegios participantes de la capital lucense

\begin{tabular}{lcc}
\hline Colegios de la capital lucense & CEIP & CPR \\
\hline Albeiros & $\mathrm{X}$ & \\
Virxe da Soidade & $\mathrm{X}$ & \\
Mercedes & $\mathrm{X}$ & \\
Anexa & $\mathrm{X}$ & \\
A Ponte & $\mathrm{X}$ & \\
Quiroga Ballesteros & $\mathrm{X}$ & \\
Sagrado Corazón & $\mathrm{X}$ & \\
\hline Rosalía de Castro & $\mathrm{X}$ & \\
Casás & $\mathrm{X}$ & \\
\hline Luis Pimentel & $\mathrm{X}$ & \\
Paradai & $\mathrm{X}$ & \\
\hline Menéndez Pelayo & $\mathrm{X}$ & \\
Illa Verde & $\mathrm{X}$ & \\
\hline Cervantes & & $\mathrm{X}$ \\
Salesianos & & $\mathrm{X}$ \\
Divino Maestro & & $\mathrm{X}$ \\
\hline Fingoy & & $\mathrm{X}$ \\
Galén & & $\mathrm{X}$ \\
\hline A Inmaculada & &
\end{tabular}

\begin{tabular}{ll} 
A Milagrosa & $\mathrm{X}$ \\
María Auxiliadora & $\mathrm{X}$ \\
\hline Franciscanos & $\mathrm{X}$ \\
San José & $\mathrm{X}$ \\
\hline Seminario Diocesano & $\mathrm{X}$ \\
\hline
\end{tabular}

\section{Materiales}

Se ha elaborado una encuesta sobre hábitos, actitudes y conocimientos acerca de las drogas, y se han analizado un total de 27.126 ítems

\section{Resultados}

Los resultados encontrados confirman que 185 jóvenes han probado el alcohol (un 22,51\% del total de los encuestados) y de ellos un $60 \%$ que cursan $5^{\circ}$ de primaria y un $70 \%$ de los que cursan $6^{\circ}$ de primaria, lo han hecho en sus propias casas (acompañados de sus familias). Se pudo constatar además, que se da un mayor consumo en menores de 10 años (el alumnado de $5^{\circ}$ de primaria que ha "probado" el alcohol, casi duplica al de $6^{\circ}$ ). Además, un $90 \%$ lo ha hecho en compañía de sus familiares. El $65 \%$ de este grupo de jóvenes afirma también que el ocio de sus padres se centra en "salir a tomar algo".

Con respecto al tabaco, del total de los encuestados, sólo un 3,9\% de los menores han probado el tabaco en estas edades. Entre ellos, un 57\% lo probó por primera vez en su propia casa y en compañía de sus familiares. Los datos muestran que el $100 \%$ de la población menor que ha probado el tabaco convive con algún fumador: en un $80 \%$ con padres y/o hermanos y en un $20 \%$ con otros familiares, siendo más influyentes en el inicio del consumo los hermanos/as, que los padres y madres.

Entre un 70-80\% del total de los encuestados, reciben de sus padres/madres una paga semanal de hasta 10 euros.

\section{Conclusiones}

Los resultados muestran como la permisividad, la banalidad, la aceptación familiar y el modelado pueden influir seriamente en las conductas y actitudes positivas ante el consumo de drogas por parte de los menores.

El trabajo de los profesores/as es básico (y a veces la única manera) para reducir o minimizar los efectos que los hábitos de consumo de las familias tengan en los menores y que éstos expresan dentro del ámbito escolar.

Se propone la labor del maestro como mediador, como una alternativa dirigida principalmente a la prevención de daños y conflictos mayores.

\section{Referencias}

Boqué, M.C. (2003). Cultura de mediación y cambio social. Barcelona: Gedisa.

Hartman, D.M. \& Chesley, G. (1997). When problems arise at school: how schools and parents can work together. NASSP Bulletin 81 (591), 81-84 
Martínez, J y Robles, L. (2001): Valores de protección ante el consumo de alcohol y tabaco en la adolescencia. Psicothema, 13 (2)., 222-228

Musitu, G., Buelga, S., Lila, M. y Cava. M. J. (2001). Familia y adolescencia. Madrid. Síntesis.

Platas, M.L. (2009): Familias, jóvenes, drogas y tiempo libre. Lugo: Concello de Lugo

Platas, M.L. et al (2010): Estado de situación de tratamiento para problemas por consumo de drogas en el ámbito local: información de 19 ciudades de Europa, América Latina y El Caribe. Coímbra, Portugal: Organización de los Estados Americanos

Platas, M.L. (2012): II Plan Municipal de Prevención de Drogodependencias de Lugo, 2012-2015. Lugo: Concello de Lugo

Platas, M.L. (2012): Jóvenes y familias ante las drogas. Lugo: Concello de Lugo

Ury, W.L. (2000). Alcanzar la paz. Diez caminos para resolver conflictos en la casa, el trabajo y el mundo. Buenos Aires: Paidós.

\section{Agradecimientos}

A todos los centros educativos públicos, privados y concertados de la capital lucense

A todos los jóvenes lucenses que han respondido a esta encuesta

A todas las familias de dichos jóvenes por aceptar su participación en esta investigación

A los profesores/as por su sensibilidad con la prevención de conflictos escolares y personales derivados del ámbito familiar y por su capacidad como mediadores. 